\title{
La Brecha digital: ¿una consecuencia más de las desigualdades sociales? Un análisis de caso para Andalucía
}

\author{
José Manuel Robles y Óscar Molina \\ IESA-CSIC \\ jmrobles@iesaa.csic.es \\ omolina@iesaa.csic.es
}

Recibido: 14.09 .2006

Aceptado: 29.05 .2007

\section{INTRODUCCIÓN. EL ACCESO DESIGUAL A LAS TECNOLOGÍAS DE LA INFORMACIÓN Y LA COMUNICACIÓN: UNA VISITA AL CONCEPTO DE BRECHA DIGITAL}

En todo proceso de modernización, el cambio genera una nueva estructura de oportunidades, pero, conjuntamente, también cabe la posibilidad de que aparezcan nuevos tipos de desigualdad social o de que, al menos, se acentúen las ya existentes. La transición hacia la Sociedad de la Información no es una excepción. En ella la información - y el conocimiento que puede generarse mediante ella - deja de ser un simple medio para tener acceso a otros bienes y se erige en un valioso recurso por sí misma (Majó, 1997). Es, precisamente, en este matiz donde puede contemplarse el paso hacia una sociedad informacional (Castells, 1998). El papel que ha pasado a jugar la información ha modificado sustancialmente diversos ámbitos de la sociedad (los procesos productivos, el mercado de trabajo, la política, elementos de la vida cotidiana, ...). Es indudable, por tanto, que la información constituye, cada vez más, un recurso en sí y que, como tal, en torno a él se origina un espacio de contraste entre quienes la tienen y quienes no, $\mathrm{y}$ entre quienes acceden a ella y quienes no lo hacen; es decir, a su alrededor se generan simultáneamente oportunidades y desigualdades. Estas desigualdades referidas al acceso a la información y al conocimiento mediante las nuevas tecnologías que lo facilitan (las TIC) se recogen bajo el concepto de brecha digital, que, según diversas fuentes, fue acuñado a mediados de los noventa en los foros donde se discutía si debían o no regularizarse las fuerzas de mercado que estaban surgiendo a raíz de la expansión de las TIC, lo que se ha dado a conocer como la 
nueva economía. La Organización para la Cooperación y Desarrollo Económico (OCDE) define la brecha digital como:

«La distancia existente entre individuos, áreas residenciales, áreas de negocios y geográficas en los diferentes niveles socio-económicos en relación a sus oportunidades para acceder a las nuevas tecnologías de la información y la comunicación, así como al uso de Internet, lo que acaba reflejando diferencias tanto entre países como dentro de los mismos».

Es esta una definición genérica del concepto, pues se recogen en ella algunas de las diferentes dimensiones de la brecha digital. Posteriormente, dichas dimensiones han sido acotadas por distintos autores a través de conceptualizaciones separadas. Así, por ejemplo, Pippa Norris (2001) evidencia la multidimesionalidad del concepto mediante la consideración de tres tipos de brechas: la global, la democrática y la social. La primera de ellas «se refiere a la diferencia en el acceso a Internet entre las sociedades industrializadas y las sociedades en vías de desarrollo». En segundo lugar, Norris da cuenta de la dimensión política y participativa de la brecha, definiendo la brecha democrática como «la diferencia entre aquellos que usan las nuevas tecnologías para participar en la vida pública y aquellos que no lo hacen». Por último, la brecha social es considerada como aquella que se da dentro de un mismo país o sociedad, como resultado de su estratificación social o geográfica interna. Este tipo de brecha digital se hace eco de las diferencias en el acceso entre distintas regiones, grupos sociales y entre individuos según su capacidad económica, su sexo, su nivel educativo, su raza o lugar de residencia (brecha geográfica).

Sin embargo, la noción de brecha digital no es tan homogénea como podría parecer (Peter y Valkenburg, 2006). Existen, en principio, diferencias reseñables en función de la concepción conceptual y teórica de este fenómeno que obligan a los académicos a optar por una postura u otra. En función del concepto de brecha digital la polémica se establece entre aquellos que asumen que las desigualdades en el uso de Internet se producen entre los que tienen y los que no tienen acceso a Internet (DiMaggio et al, 2001) y entre aquellos otros que definen las desigualdades no solamente como resultado del acceso potencial a Internet sino también como las diferencias en el uso de esta tecnología (Bucy, 2000. Van Dijk, 2002). Las diferencias en un sentido teórico se establecen, por su parte, entre aquellos que entienden que la brecha digital es una expresión más de las diferencias sociales existentes en un determinado territorio y que, por lo tanto, dependen de las características sociodemográficas de dicho territorio (Van Dijk y Hacker, 2003), y entre aquellos que definen el carácter original y distintivo de la brecha digital como consecuencia de las relaciones entre ciudadanos y tecnologías (Anderson et al, 2001. Compaine, 2001). Entre estos últimos, existe el convencimiento de que la extensión de Internet hará posible la desaparición de la brecha digital y permitirá «el triunfo de la descentralización, la globalización, la armonización y el empoderamiento» (Negroponte 1995, 
229). Mientras, entre los primeros se mantiene una concepción mucho mas pesimista según la cual las diferencias en el acceso y uso de las TIC disminuirán en la misma medida en que lo hagan las diferencias sociales y estructurales existentes.

Una de las principales conclusiones que han obtenido los estudios sobre la brecha digital ha sido la importante influencia que ejercen la variable hábitat y las variables geográfico-políticas (provincia, región o nación) sobre el nivel de acceso y uso de las TIC (Robles, 2005). En términos más prosaicos, podríamos decir que existe un importante volumen de estudios que muestran cómo el lugar en el que reside el ciudadano se transforma en una circunstancia que determina sobre manera el acceso y uso de las TIC. Sin embargo, y pese al creciente número de estudios sobre la brecha digital y, concretamente, sobre la brecha digital de carácter geográfico, aún restan muchas e importantes cuestiones por zanjar. En este artículo, nos hemos propuesto como objetivo, analizar una de estas cuestiones, a saber, ¿en qué medida la brecha digital geográfica determina las diferencias en el equipamiento y uso de las TIC? Concluiremos que no es posible atribuir las diferencias en el uso de Internet a variables geográficas al uso, es decir, a las variables tamaño de Hábitat y organización geográfico-política. Mantendremos, por el contrario, que la brecha digital geográfica, tal y como es entendida por los estudios sobre brecha digital y por los estudios sobre Sociedad de la Información, es reducible a la brecha digital social. Igualmente propondremos la utilización de una variable geográfica que atiende a patrones distintos a los que se definen en la variable Hábitat y en la variable geográfico-política y que definimos en este estudio como «zona geográfica» ${ }^{1}$.

De este modo, nuestro estudio se enmarca en la polémica en torno a la brecha digital al tratar de mostrar cómo, en términos generales, las diferencias en el uso de Internet en Andalucía son explicables en función de las diferencias sociodemográficas existentes en el territorio y, en términos más concretos, mostrando como una de las más importantes variables que influyen sobre la brecha digital, a saber, la de carácter geográfico, es también reducible a este tipo de variables. Este resultado apoya la tesis de Van Dijk y Hacker (2003) al reducir el factor territorial a un factor social y, por lo tanto se posiciona bajo la tesis de que la brecha digital no disminuirá sensiblemente hasta que no lo hagan las diferencias sociales que la producen. Es importante destacar, por último, que, en principio, consideramos la brecha digital como la diferencia entre aquellos que usan y aquellos que no usan Internet. Nos decantamos por esta opción al considerar que la definición de brecha digital en función de los usos de Internet es una concepción asociada a una fase de evolución de la Sociedad de la Información más avanzada. Dadas las importantes diferencias existentes entre aquellos que usan y aquellos que no usan Internet, la extensión de la definición a las diferencias en función del uso de Internet es aún prematura.

${ }^{1}$ Esta variable está definida por las siguientes cuatro categorías: zona interior, zona urbana, zona turística y zona de agricultura intensiva.

EMPIRIA. Revista de Metodología de Ciencias Sociales. N. ${ }^{\circ}$ 13, enero-junio, 2007, pp. 81-99.

ISSN: $1139-5737$ 
Para cumplir con los objetivos de este estudio, procederemos de la siguiente forma. En el primer apartado, trataremos de definir de manera más concreta qué es la brecha digital y, más específicamente, qué entendemos como brecha digital geográfica, así como su medida concreta para el caso de Andalucía. Para ello haremos uso de la serie de estudios I@landalus realizados por el Instituto de Estudios Sociales Avanzados (IESA-CSIC) por encargo de la Consejería de Innovación, Ciencia y Empresa de la Junta de Andalucía ${ }^{2}$. En segundo lugar, ofrecemos una herramienta metodológica que nos ayuda a definir y controlar el papel de este tipo de brecha en el uso de las Tecnologías de la Información y la Comunicación. En tercer lugar, presentaremos una reelaboración del concepto de brecha digital bajo la luz de los resultados obtenidos a partir de este método estadístico para, por último, ofrecer algunas conclusiones sobre las implicaciones de nuestro modelo. Nuestro objetivo general es analizar, en primer lugar, el caso andaluz para, en futuras investigaciones, avanzar, mediante estudios comparativos, en el conocimiento de las distintas vertientes de la brecha digital en España.

\section{LA BRECHA DIGITAL EN CIFRAS: EL CASO DE LA SOCIEDAD DE LA INFORMACIÓN EN ANDALUCÍA}

La Sociedad de la Información ha experimentado en Andalucía una importante evolución durante los últimos años. El aumento en el equipamiento en los hogares andaluces de las tecnologías tradicionalmente relacionadas con la Sociedad de la Información así como el aumento en el uso de las mismas ha sido enormemente significativo. Los hogares que contaban con ordenador en 2002 apenas superaban el $30 \%$ del total de hogares andaluces. Mientras, en 2005 el $43,5 \%$ los hogares de esta comunidad están equipados con ordenador. Un caso muy similar encontramos en el porcentaje de hogares con acceso a Internet. En 2002, el porcentaje de hogares andaluces con acceso a Internet rondaba el 14\%. Por su parte, en 2005 este porcentaje era superior al $25 \%$.

Según los datos de los Informes I@landalus podemos afirmar que la evolución en el uso de estas tecnologías es también positiva. El porcentaje de andaluces que utilizaban el ordenador en 2002 era del 40\%, tres años más tarde, en 2005, este porcentaje había ascendido hasta alcanzar el 51,2\%. Esto significa que, en dicho año, el número de andaluces usuarios de ordenador era de 3.242.318. Por su parte, el aumento de los usuarios andaluces de Internet es también significativo al experimentar un incremento de más de diez puntos porcentuales. En 2005 el número de Internautas en Andalucía alcanzaba el 40,2\% de la población o, lo que es lo mismo, 2.541 .747 ciudadanos.

\footnotetext{
${ }^{2}$ Los estudios I@landalus llevan realizándose desde el año 2002 y su objetivo es la medición de la evolución así como del estado de la Sociedad de la Información en Andalucía. Con un total de 5.520 encuestas presenciales realizadas a hogares e individuos con una edad de 14 o más años de edad, los estudios I@landalus recogen un total de 71 indicadores de equipamiento y uso de telefonía móvil, ordenador e Internet así como sus distribuciones sociodemográficas y geográficas.
} 


\section{La brecha digital social en Andalucía}

Pese a estas cifras, la distribución del equipamiento y el uso de las TIC en Andalucía es bastante irregular. La variable sociodemográfica que determina en mayor medida la tenencia de Internet en los hogares andaluces es la clase social. Otra de las variables más importantes es el tamaño del hogar. Es decir, cuanto más miembros constituyen el hogar, mayor es la probabilidad de que cuenten con esta tecnología. Entre los hogares de cuatro y los hogares de una persona la diferencia es, en 2005, de 28 puntos. De entre los hogares de mayor tamaño (tres o más miembros) son los hogares de clase media y alta los que presentan mayores porcentajes de acceso a Internet. Así, el 58\% de los hogares de este tipo están equipados en 2005 con acceso a Internet. Esto significa que prácticamente duplican la media andaluza $(25,8 \%)$.

El uso de Internet también presenta una distribución irregular. Las mayores diferencias en el uso de esta TIC se expresan, tal y como muestran los gráficos 1 y 2 , en función de la edad y del nivel de estudios de los ciudadanos. Así, encontramos como entre los más jóvenes (personas entre 14 y 19 años) el porcentaje de usuarios de Internet asciende hasta el 85,2\%. Mientras, entre las personas entre 55 y 64 años este porcentaje apenas alcanza el $10 \%$.

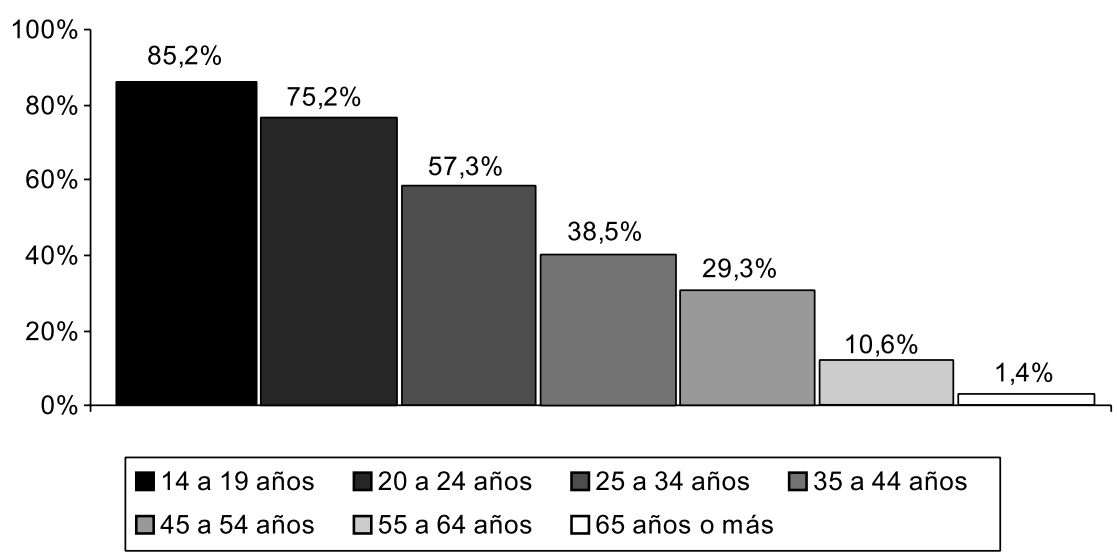

Gráfico 1. Uso de Internet según edad.

Según la variable «nivel educativo» las diferencias son también muy significativas. Tal y como vemos en el gráfico siguiente, los ciudadanos con estudios universitarios que utilizan Internet son el 82,3\% de los ciudadanos con este nivel educativo. En el lado opuesto, solo un cuarto de los ciudadanos con estudios primarios es usuario de esta tecnología.

Estas diferencias (brecha digital social) son aún más patentes si comparamos los segmentos poblacionales en los que existe un mayor porcentaje de usuarios 


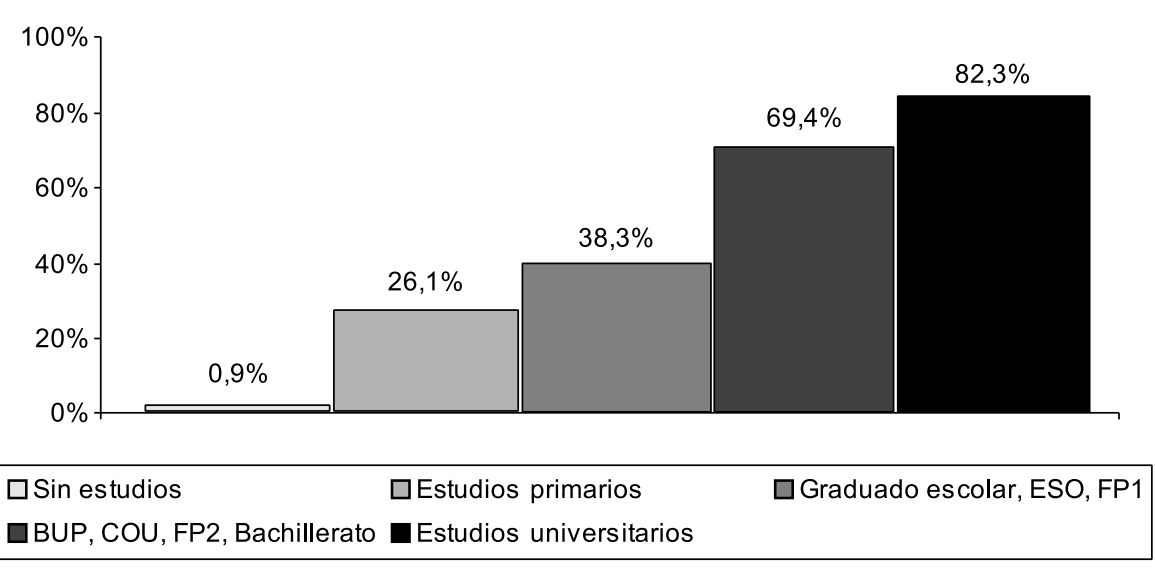

Gráfico 2. Uso de Internet según nivel educativo.

con los segmentos en los que hay menos usuarios. Así, son las personas de hasta 34 años con estudios superiores los que en mayor medida utilizan esta tecnología. Más de nueve de cada diez ciudadanos andaluces con estas características son usuarios (91\%). El porcentaje de usuarios de Internet es también muy alto entre las personas en edad escolar (hasta 18 años y con estudios de primaria o secundaria). Entre estos, el 85\% utilizó Internet durante el último año. Mientras, en el polo opuesto, las personas mayores de 35 años con niveles de estudios inferiores al bachillerato son los que en menor medida utilizan Internet (18\%). Esta situación se agrava sobremanera entre las personas de este perfil que se encuentran inactivos $(0,9 \%)$.

El aumento de usuarios de Internet en Andalucía no se ha visto acompañado de una reducción de la denominada brecha social. Las diferencias entre aquellos grupos sociales más retrasados en la incorporación al uso de Internet y aquellos otros más avanzados se mantienen prácticamente igual que en 2002. En aquel año, un $11 \%$ más de hombres que de mujeres hacía uso de esta tecnología. En 2005 esta diferencia continúa estando en torno a 12 puntos porcentuales. Las diferencias en el uso de Internet en función del sexo siguen siendo, por lo tanto, muy importantes en Andalucía (46,2\% de usuarios por 34,4\% de usuarias en 2005). Sin embargo, estas diferencias son especialmente significativas cuando se unen a las situaciones de desempleo y/o a niveles de estudios inferiores al bachillerato. Cuando estas circunstancias se producen la brecha según sexos alcanza los 20 puntos porcentuales. La misma situación se produce cuando hablamos de brecha social según edad.

En resumen, la población más adulta, las mujeres, las personas con más bajo nivel educativo, los pensionistas, las amas de casa, las personas incapacitadas, $\mathrm{y}$ quienes tienen una capacidad económica baja siguen, comparativamente, quedando al margen de la Sociedad de la Información de forma similar a 2002. Esto 
no hace sino señalar los grupos de población hacia los cuales han de orientarse de manera especial las políticas que se lleven a cabo en el marco «civil» de la Sociedad de la Información en Andalucía (políticas de e-inclusión).

\section{La brecha digital geográfica en Andalucía}

Junto con el análisis en función de variables sociodemográficas, el estudio de la brecha digital, ha atendido también a las diferencias según variables geográficas (Robles, 2005). Además del interés meramente científico, el estudio de la brecha digital en función de las variables geográficas tiene también un importante cariz político. Esto se debe a que las Administraciones Públicas, tanto regionales como nacionales, han vertebrado, en gran medida, sus políticas de disminución de la brecha digital sobre la base de la organización geográfica y geográfico-política de la población. De esta forma, el análisis de las diferencias en el acceso y uso de Internet es especialmente relevante debido, no sólo a cuestiones científicas, sino que, también, a su importancia para la implementación de un gran número de políticas públicas ${ }^{3}$. En lo que sigue analizaremos la brecha digital geográfica en Andalucía.

En los estudios I@landalus hemos atendido a dos tipos principales de variables geográficas; provincia y tamaño de hábitat. La variable provincia se configura como una variable geográfico-política que agrupa a la población en función de la demarcación administrativa. Por su parte, la variable tamaño de hábitat es una variable en la que se agrupan las poblaciones andaluzas en función del número de habitantes que residen en ellas. Tal y como muestra el mapa 1 y el gráfico 3 la distribución del uso de Internet según estas variables es significativamente irregular. Así, en el caso del uso de Internet según provincia encontramos que entre las provincias más avanzadas en el uso de Internet y las provincias con un menor porcentaje de usuarios las diferencias alcanzan más de diez puntos porcentuales. Así, mientras que en la provincia de Málaga el porcentaje de usuarios de Internet alcanzó en 2005 el 44,6\%, en la provincia de Jaén el porcentaje apenas alcanza el $30 \%$.

También encontramos diferencias apreciables según la variable tamaño de hábitat. La brecha digital existente entre los municipios andaluces mayores de 50.000 habitantes y los municipios andaluces menores de 5.000 habitantes superan los 12 puntos porcentuales. Esto significa que existe una importante brecha digital en Andalucía en función de las variables geográficas. Esta diferencia privilegia a un conjunto de provincias andaluzas sobre otras, principalmente a las provincias de Málaga, Granada y Cádiz por encima de las provincias de Jaén y Huelva, y a las poblaciones más habitadas sobre las poblaciones menos habitadas.

${ }^{3}$ En el caso de Andalucía, una de las más importantes políticas de disminución de la brecha digital, el Programa Guadalinfo, está orientado a la promoción del equipamiento y uso de las TIC en los municipios andaluces menores de 10.000 habitantes.

EMPIRIA. Revista de Metodología de Ciencias Sociales. N. ${ }^{\circ}$ 13, enero-junio, 2007, pp. 81-99.

ISSN: $1139-5737$ 


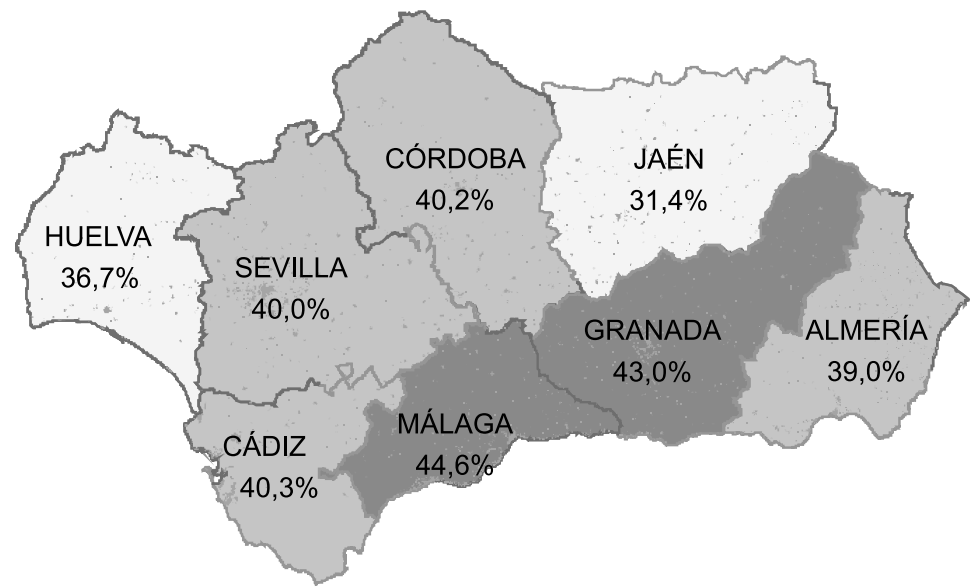

Por encima de la media Igual a la media

Por debajo de la media

Mapa 1. Uso de Internet según provincias.

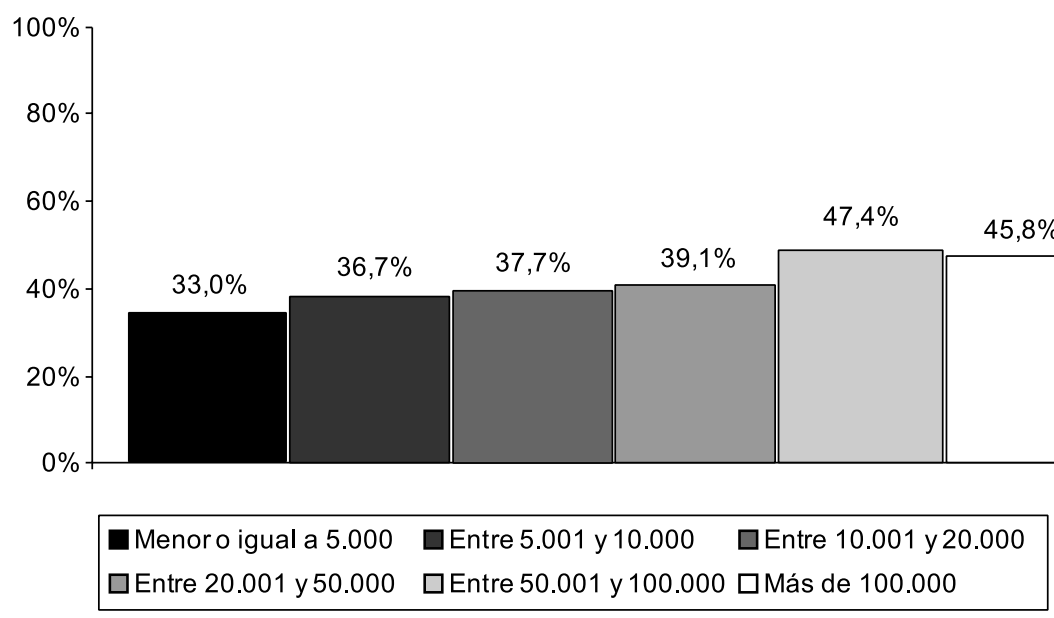

Gráfico 3. Uso de Internet según tamaño de hábitat.

Tal y como mencionamos en la introducción, en este estudio, nos propusimos investigar la naturaleza de la brecha digital geográfica así como las razones por las que unas determinadas provincias y unos determinados tamaños de hábitat presentaban mayores niveles de equipamiento y uso de TIC y, más concretamente, de uso de Internet. Nuestra principal hipótesis de partida incidía en la idea de que las diferencias geográficas apreciables en un análisis descriptivo se enraizaban en as- 
pectos más profundos de la estructura social. Los datos muestran, tal y como ha sido expuesto aquí, que, por ejemplo, existen provincias andaluzas como Málaga en las que el porcentaje de usuarios de Internet llega a superar la media española de usuarios de estas tecnologías mientras que en otras provincias, como Jaén, este porcentaje es notablemente inferior a la media andaluza. Dados estos resultados, nos preguntamos si las provincias de, por ejemplo, Málaga y Jaén presentan características geográficas singulares que expliquen su situación polarizada en el uso de esta tecnología o si, por el contrario, estas diferencias se deben a la distinta conjunción de variables sociales que están presentes en dichas provincias. En otras palabras, ¿la brecha digital geográfica queda explicada, tal y como muestran los primeros análisis, por las variables tamaño de hábitat y provincia o se debe a que en determinados territorios existen más ciudadanos con determinadas características asociadas a la no utilización de Internet?

Para responder a esta cuestión, desarrollamos un modelo estadístico que nos permitió analizar la influencia de las variables geográficas sobre la variable uso de Internet así como el peso de las variables sociodemográficas sobre las variables geográficas. En lo que sigue mostraremos este mecanismo metodológico así como los primeros resultados obtenidos.

\section{EL MÉTODO}

En este apartado y mediante técnicas de análisis multivariante, como regresión logística y segmentación, se tratará de comprobar cómo influyen aspectos como la edad o el nivel de estudios en el hecho de ser usuario de Internet, y cómo a partir de las distintas estructuras sociodemográficas de cada provincia y de cada hábitat se puede dar explicación a las diferencias que presentan en porcentajes de usuarios de Internet. En primer lugar, se ha realizado una regresión logística, herramienta analítica de gran utilidad cuando se trata de comprobar si varias variables independientes determinan la ocurrencia o no de un determinado evento, en este caso, el uso de Internet. Así pues, la variable dependiente es el uso o no de Internet (0: «no lo usa» y 1: «lo usa»), y las variables independientes introducidas en el modelo son: edad, sexo, estado civil, nivel de estudios, situación laboral, tamaño de hábitat, tamaño de hogar, clase social, actividad del cabeza de familia, zona geográfica y tipo de barrio.

El proceso seguido, parte de la introducción de la variable dependiente que se desea ajustar, a saber, usuarios de Internet, y las independientes a partir de las cuales se quiere explicar el comportamiento de dicha variable que son, en este caso, las variables sociodemográficas. El método elegido para la inclusión de variables independientes en el modelo es «condicional hacia delante», de forma que sea lo más parsimonioso posible, es decir, el modelo que permita con el menor número de variables independientes hacer buenas «predicciones» de la variable dependiente. Entre los resultados obtenidos a partir de este modelo de regresión destacan diversas medidas sobre la bondad del ajuste, variables 
independientes que forman parte del modelo (están relacionadas significativamente con la variable dependiente), variables independientes que se quedan fuera del modelo, probabilidad de ser usuarios de Internet según la categoría de cada variable independiente incluida en el modelo a la que se pertenezca...etc. Además, para cada persona de la muestra, se obtiene a partir de dichas variables independientes la probabilidad de ser usuario de Internet y las diferencias o residuos de estas probabilidades teóricas con los valores reales observados (1 si son usuarios, 0 si no lo son). A continuación se repite el mismo proceso, pero añadiendo la variable «provincia» como independiente. Con esto se persigue comprobar la suficiencia de las variables independientes para explicar el comportamiento de la variable dependiente, es decir, se trata de saber si las variables sociodemográficas determinan por sí solas el uso de Internet o, por el contrario, existen otras variables no controladas en el estudio que influyen en dicho uso. Por tanto, si el modelo resultante incluye la variable provincia como significativa para la determinación de la variable dependiente, la conclusión será que existen otras variables, además de las variables sociodemográficas, que influyen en la variable dependiente. Si, por el contrario, la variable «provincia» queda fuera del modelo, quiere decir que las variables sociodemográficas son suficientes para explicar la variable dependiente, luego es posible estudiar las diferencias de usuarios de Internet entre las distintas provincias de Andalucía a partir de la estructura sociodemográfica de las mismas.

Se ha comentado anteriormente que uno de los resultados obtenidos con la regresión logística son las predicciones (probabilidad de uso de Internet) y los residuos (diferencia entre valor teórico y valor observado) para cada individuo de la muestra. Si se calcula la media por provincias de esta variable «predicción», el resultado será la probabilidad media por provincias de ser usuario de Internet o, dicho de otra forma, la proporción teórica (se obtiene a partir de las variables sociodemográficas incluidas en el modelo) de usuarios de Internet para cada provincia. Es obvio pensar que si el modelo de regresión obtenido ajusta bien la variable dependiente a partir de las independientes, esta proporción teórica se acercará bastante a la proporción real observada (muestral) en cada provincia, luego la media de los residuos por provincia será próxima a cero. Este resultado permitirá estudiar si el modelo proporciona buenos ajustes por provincias mediante un contraste de hipótesis sobre la media de los residuos en cada una de ellas. Si en una provincia se acepta la hipótesis de que la media de los residuos es cero, entonces estará bien ajustada por el modelo, en caso contrario, obtendremos malas predicciones de la variable dependiente a partir de las variables independientes, lo que quiere decir que las variables sociodemográficas no determinan por sí solas el uso de Internet en dicha provincia. Luego, independientemente de que la variable «provincia» esté incluida o no en el modelo, es posible analizar para qué provincias se obtienen buenas predicciones de uso de Internet a partir de las variables sociodemográficas que forman parte del modelo y estudiar las diferencias de estas provincias, en cuanto a porcentaje de usuarios, a partir de la estructura sociodemográfica de las mismas. 
El siguiente paso será contrastar qué provincias difieren significativamente en porcentaje de usuarios respecto a la media de Andalucía (tomada como valor crítico) y, si estas provincias están bien ajustadas por el modelo, estudiar qué características estructurales de las mismas pueden influir en estas diferencias. Por último, como complemento a lo obtenido, se ha realizado una segmentación con el objetivo de encontrar qué combinaciones de categorías de estas variables sociodemográficas son las más proclives al uso de Internet.

\section{Principales resultados}

El modelo teórico de regresión obtenido permite afirmar que el uso de Internet está muy relacionado con las características sociodemográficas de los individuos ${ }^{4}$, sobre todo con la edad, el nivel de estudios, la clase social a la que pertenecen y la situación laboral en la que se encuentran. El resto de variables sociodemográficas, exceptuando tamaño de hábitat, también influyen de forma significativa en el uso de Internet. Así pues, son los más jóvenes los que en mayor medida utilizan Internet, disminuyendo el porcentaje de usuarios a medida que aumenta la edad. También se observa una mayor concentración de usuarios en aquellos estratos con un mayor nivel de estudios, así como en las capas sociales que cuentan con más recursos económicos.

La bondad del ajuste del modelo se ha confirmado a partir de un análisis de residuos en el que se ha contrastado la proximidad de los valores teóricos (probabilidad media de ser usuario) a los observados (proporción de usuarios observada) en cada provincia ${ }^{5}$. Esto significa que a partir de las variables sociodemográficas se pueden hacer «buenas» predicciones por provincias del porcentaje de usuarios de Internet en las mismas.

Cuando se repite el proceso añadiendo la variable «provincia» como independiente, ésta no entra en el modelo, esto es, no es significativa en la determinación de los usuarios de Internet. Esto no quiere decir que no existan diferencias significativas de usuarios entre las distintas provincias (de hecho existen), sino que tales discrepancias se pueden explicar a partir de las variables sociodemográficas contempladas en el modelo. La principal consecuencia que se deriva de este resultado es que las diferencias de usuarios de Internet entre provincias no se deben al hecho de pertenecer a una determinada zona geográfica entendida como delimitación administrativa, sino a otras variables cuya distribución es desigual en dichas provincias. Sin embargo, la variable «zona» ${ }^{6}$, que establece una división ge-

${ }^{4}$ Existe una elevada correlación de la variable dependiente con las independientes $\left(\mathrm{R}^{2}\right.$ de Nagelkerke $=0,685$ ). A partir de las variables sociodemográficas se clasifican bien al $85,9 \%$ de los casos.

${ }^{5}$ En todos los contrastes por provincias se acepta la hipótesis nula de que la media de los residuos es cero.

${ }^{6}$ Esta variable consta de cuatro categorías: zona interior, zona urbana, zona turística y zona de agricultura intensiva.

EMPIRIA. Revista de Metodología de Ciencias Sociales. N. ${ }^{\circ}$ 13, enero-junio, 2007, pp. 81-99.

ISSN: $1139-5737$ 
ográfica atendiendo a la actividad principal del lugar y su ubicación, si aparece en el modelo como significativa, siendo la única de las tres variables geográficas consideradas (provincia, tamaño de hábitat y zona) en la que las diferencias de usuarios entre las distintas categorías (mayor porcentaje de usuarios en zonas urbanas y turísticas que en zonas de agricultura intensiva y de interior), no se pueden explicar a partir del resto de variables independientes.

Una vez que se ha especificado el modelo, se ha comprobado la bondad del ajuste y se han indicado las variables más significativas, el siguiente paso será tratar de dar explicación a las diferentes cifras de usuarios que presentan las provincias andaluzas. Para ello, se ha analizado como se distribuyen las principales variables que influyen en el uso de Internet en las provincias que presentan mayores diferencias porcentuales respecto a la media de Andalucía, a saber, Málaga y Granada con los niveles más altos de usuarios (44,6\% y $43 \%$ respectivamente), Jaén y Huelva con los más bajos (31,4\% y 36,7\% respectivamente).

Jaén es la provincia que presenta un menor porcentaje de personas con una edad comprendida entre 14 y 40 años (46,8\% frente al 50,7\% de los casos totales), mientras que el porcentaje de personas mayores de 60 años en esta provincia supera notablemente a la cifra obtenida cuando se consideran todos los casos (26,5\% frente al 21,4\% de los casos totales). Por tanto, Jaén es la provincia más envejecida de Andalucía, y esto puede suponer una de las causas de un menor porcentaje de usuarios de Internet en esta provincia, ya que, como se ha comentado anteriormente, los más jóvenes son más proclives al uso de Internet.

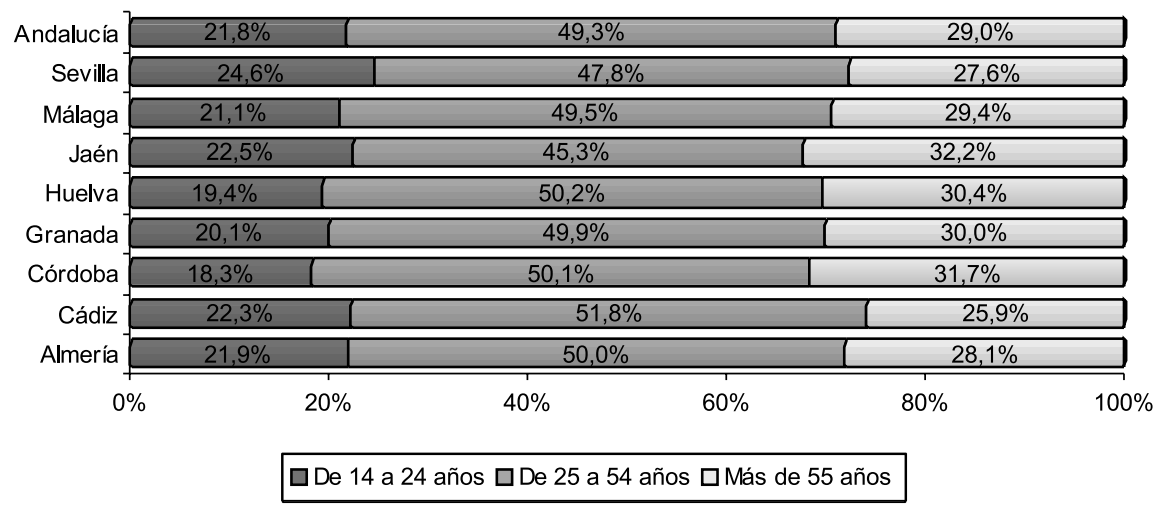

Gráfico 4. Distribución provincial según edad.

Analizando el nivel de estudios de los encuestados por provincias, se aprecian diferencias importantes en las cifras de personas con estudios universitarios que presentan Granada y Málaga (15,5\% y $14,1 \%$ respectivamente), y las que presentan Jaén y Huelva $(10,3 \%$ y $8,1 \%)$. A esto hay que añadir que Jaén es la provincia con un mayor porcentaje de encuestados sin estudios $(24,7 \%$ frente al 
$17,6 \%$ del total de casos). Esto también puede ser una de las causas de las diferencias en las cifras de usuarios de Internet de las que se ha hablado, ya que se observa una mayor presencia de éstos en los grupos con un mayor nivel de estudios.

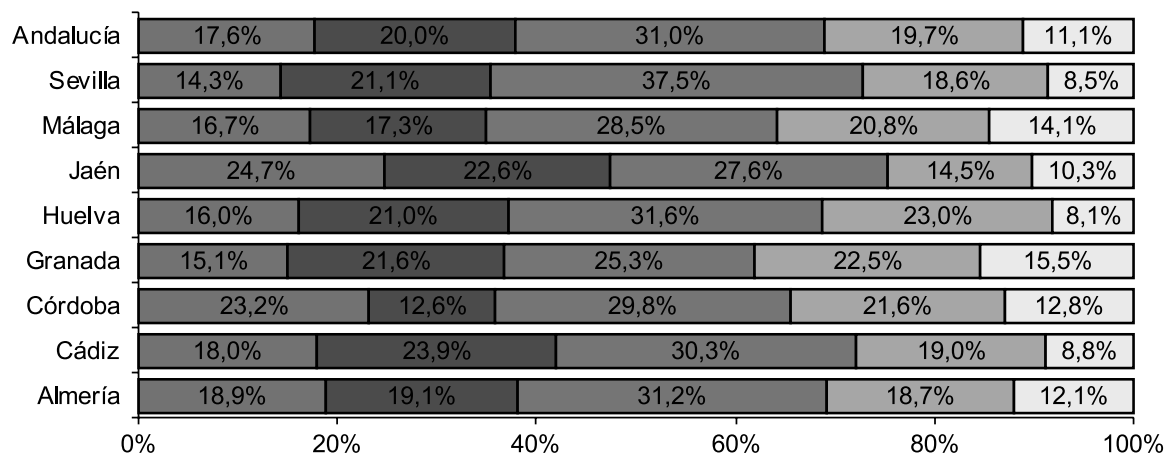

Sin estudios $\square$ Estudios primarios $\square$ Graduado escolar, ESO... $\square$ BUP, COU... $\square$ Estudios universitarios

Gráfico 5. Distribución provincial según nivel de estudios.

Otra variable relacionada con el uso de Internet es la clase social que, como en anteriores casos, presenta una distribución distinta en estas provincias. Así pues, en Granada el porcentaje de personas de clase media y alta asciende al $43,6 \%$ y en Málaga esta cifra se acerca al 40\%, mientras que en Jaén apenas superan el 30\% y en Huelva sólo suponen el 27,8\%. De la misma forma, los grupos de clase baja están más presentes en estas dos últimas provincias que en las dos primeras. Estas diferencias socioeconómicas también pueden ser uno de los motivos por los cuales los porcentajes de usuarios en estas provincias son tan distintos, pues se ha comprobado que en los estratos que tienen más recursos económicos el porcentaje de usuarios de Internet es muy superior que en los más desfavorecidos.

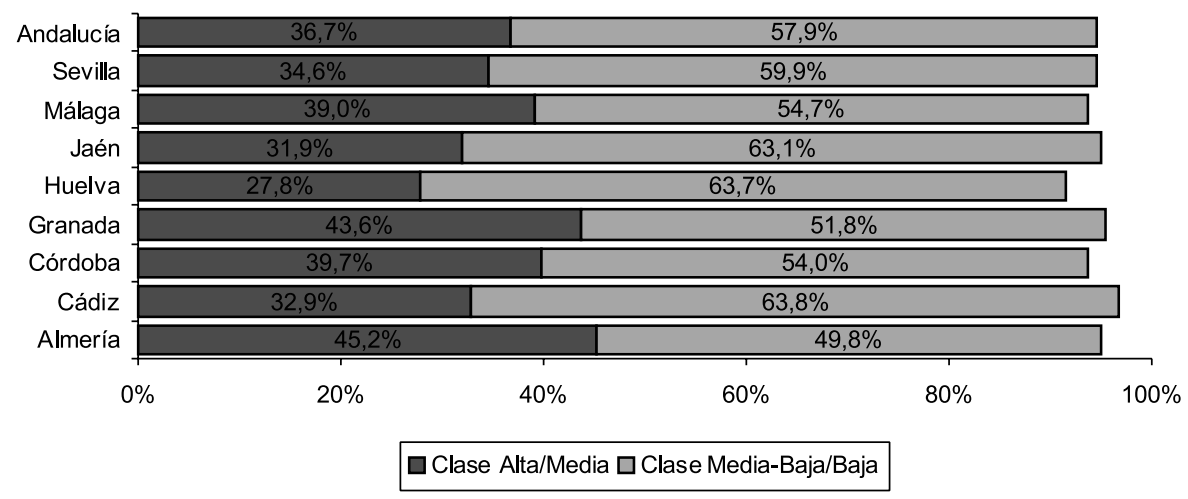

Gráfico 6. Distribución provincial según clase social. 
La conclusión a la que se llega después de haber analizado las variables que están más relacionadas con el uso de Internet en las provincias que presentan mayores discrepancias respecto a la media de Andalucía, es que tales diferencias se pueden explicar a través del estudio de las diversas estructuras sociodemográficas de estas provincias, que propician la concentración de usuarios en unos casos más que en otros. Los resultados obtenidos a partir del análisis de segmentación confirman y complementan los ya comentados, obteniéndose como variables más discriminantes del uso de Internet la edad, el nivel de estudios y la situación laboral. La ventaja de esta técnica es que proporciona las combinaciones de categorías de estas variables (segmentos) en las que se encuentran las mayores y las menores concentraciones de usuarios. Los segmentos con mayor porcentaje de usuarios son los formados por jóvenes con estudios avanzados, mientras que los formados por adultos sin estudios o con un nivel bajo de estudios presentan las cifras más bajas de usuarios.

\section{IMPLICACIONES SOCIALES DEL PESO DE LAS VARIABLES SOCIODEMOGRÁFICAS SOBRE LA BRECHA DIGITAL GEOGRÁFICA}

Para las administraciones públicas y más concretamente para las administraciones públicas que gestionan el área relacionada con las tecnologías 7 , la reducción de la brecha digital se ha transformado en una de sus prioridades. Tanto la administración central como la mayor parte de las administraciones regionales tienen en marcha iniciativas orientadas a la reducción de las diferencias en el equipamiento y uso de Internet entre los ciudadanos de sus distintas comunidades. Este es el caso del Programa CAPI de la Comunidad de Madrid, el programa Disemina de la Comunidad Valencia, el Plan Konekta Gunea del Gobierno Vasco o de la iniciativa Guadalinfo promovida por la Junta de Andalucía. Uno de los ejes principales sobre los que se vertebran estas iniciativas es la organización geográfica de dichas comunidades. Así, por ejemplo, la iniciativa Guadalinfo está orientada a la difusión del uso de Internet a través de banda ancha a los municipios andaluces menores de 10.000 habitantes. Estas iniciativas privilegian, pues, la importancia de la disminución de la brecha digital geográfica por encima de otros tipos de brecha digital tales como la brecha digital social.

En este trabajo hemos mostrado como la variable geográfica es una variable reducible a las variables sociales que introdujimos en muestro modelo estadístico. Este hecho pone de manifiesto algunas cuestiones. En primer lugar, para la explicación de las razones de las diferencias en el uso de Internet entre unos ciudadanos y otros, las variables geográficas (provincia y tamaño de hábitat) no

${ }^{7}$ En el caso del estado español nos referimos al Ministerio de Ciencia y Tecnología y en el caso de Andalucía nos referimos a la Consejería de Innovación, Ciencia y Empresa de la Junta de Andalucía. 
ofrecen información que no esté contenida en las variables sociales introducidas en el modelo. En este sentido, afirmamos que las diferencias anteriormente señaladas que afectan a la distribución geográfica del uso de Internet en Andalucía son achacables a las diferencias en la distribución social de dichas zonas geográficas y no a alguna característica singular de dichas zonas. En otras palabras, las razones detrás de las diferencias en función del tamaño del hábitat y de las provincias andaluzas son de carácter social.

Esta importante conclusión nos permite introducir dos cuestiones relevantes. Una de carácter teórico y otra relativa a la evaluación de políticas públicas. En un sentido analítico, nuestro trabajo refuerza la idea de que la brecha digital es una forma más de las diferencias sociales que estructuran la sociedad andaluza. Nuestra tesis es que la reducción de las variables geográficas a las variables sociales demuestra que son este tipo de variables las que determinan en mayor medida la brecha digital. Así, la brecha digital se transforma en un tipo más de desigual en línea con las desigualdades económicas y sociales. Tal y como han mostrado Marquez y Selbor (2004) la existencia de grandes diferencias sociales en un determinado territorio dificultará sino impedirá el desarrollo de la Sociedad de la Información más allá de aquellos grupos sociales con mayores niveles de sensibilización hacia las tecnologías, a saber, las personas jóvenes con estudios superiores y con ingresos por encima de la media. En la línea de autores como Van Dijk y Hacker (2003) manifestamos, pues, que la reducción de la brecha digital está supeditada de una forma muy importante a la reducción de diferencias sociales tales como las de carácter educativo o las relativas al nivel de ingresos. En este sentido, con este trabajo, hemos tratado de aportar un caso de estudio más en el que se muestra la capacidad explicativa de este conjunto de hipótesis.

En el plano de la evaluación de las políticas públicas, nuestros resultados ponen de manifiesto que políticas sectoriales orientadas al fomento del uso de Internet entre grupos poblacionales concretos en función de variables como la edad, el nivel educativo o la clase social es una estrategia política que podría reportar, en general, mejores resultados que los obtenidos a través de políticas de reducción de la brecha digital en función de las zonas geográficas. El Instituto de Estudios Sociales Avanzados (IESA-CSIC) ha realizado el seguimiento de una de las principales políticas de reducción de la brecha digital en Andalucía; el Proyecto Guadalinfo. Tal y como se ha mencionado más arriba, este proyecto está dirigido a la difusión del uso de Internet en los municipios andaluces menores de 10.000 habitantes al considerarse ${ }^{8}$ este tipo de entorno geográfico más proclive a quedar retrasado en la incorporación a la Sociedad de la Información. Ciertamente, y pese a haberse experimentado un ascenso significativo en el uso

${ }^{8}$ Esta consideración no es arbitraria. Queda avalada por los resultados de los indicadores descriptivos de carácter geográfico del tipo que hemos mostrado aquí. Sin embargo, tal y como tratamos de mostrar, este tipo de indicadores no ofrecen sino una explicación epidérmica de un fenómeno que consideramos mucho más profundo. 
de Internet en este tipo de municipios tras un corto espacio de tiempo 9 , no podemos decir que a día de hoy se esté produciendo un proceso de disminución de la brecha digital existente entre los municipios andaluces menores de 10.000 habitantes y el resto de municipios andaluces. Este hecho implica una importante limitación para la iniciativa, entre otras razones, ya que no se ven reflejados en términos medibles la importante inversión económica que el Gobierno Andaluz ha realizado para la reducción de la brecha digital. Nuestra contribución en este sentido consistiría en poner de manifiesto el hecho de que la implementación de políticas más sociales para la reducción de la brecha digital puede redundar en mejores resultados dados los objetivos propuestos por la administración.

Por último, nos gustaría poner de manifiesto que si se considera oportuna la utilización de una variable geográfica para las políticas de reducción de la brecha digital, la variable más adecuada es la que denominamos en este estudio como «zona geográfica». Tal y como muestran los resultados de nuestro modelo, la variable zona geográfica no es reducible explicativamente a ningún conjunto de variables sociales. Según nuestro modelo, la variable «zona geográfica» se distinguía del resto de variables geográficas al no ser explicada completamente por las variables sociales lo cual significa que introduce información singular sobre algún aspecto de la realidad geográfica andaluza. Como hemos dicho, en esta variable se encuentran agrupadas un conjunto de zonas en función de su actividad económica y su ubicación. Así, existen cuatro categorías; zona interior, zona urbana, zona turística y zona de agricultura intensiva. En principio, es razonable suponer que el hecho de que en esta variable se aúnen, junto con variables geográficas, variables de tipo político y económico dota a «zona geográfica» de unas características no reducibles a las meras variables sociales y que la convierten una herramienta de gran utilidad para medir las diferencias en el uso de Internet. En este sentido, mantenemos que se trata de la variable más informativa si se desea realizar una tabulación geográfica ya que, a diferencia de las variables provincia o tamaño de hábitat, su información no es recogida por la conjunción de otro tipo de variables.

\section{CONCLUSIÓN}

Hemos pretendido que nuestro trabajo se uniera a esta serie de investigaciones que tratan de poner de manifiesto la naturaleza y riesgos de la brecha digital. Nuestro objetivo ha sido reforzar la idea de que la brecha digital es una consecuencia más de las desigualdades sociales que estructuran las sociedades modernas. En este sentido, hemos mostrado cómo las diferencias en el uso de Internet son reducibles a diferencias estructurales tales como la edad de los

${ }^{9}$ El inicio del programa Guadalinfo se realizó en el año 2003. Por esta razón, sólo contamos con información sobre la evolución de la Sociedad de la Información en estos municipios correspondiente a los años 2004 y 2005.

EMPIRIA. Revista de Metodología de Ciencias Sociales. N. ${ }^{\circ}$ 13, enero-junio, 2007, pp. 81-99. ISSN: $1139-5737$ 
ciudadanos, la clase social a la que pertenecen o el nivel de estudios con los que cuentan. De igual forma hemos tratado de mostrar cómo incluso una de las variables no sociales más destacadas en la polarización del uso de Internet, la brecha digital geográfica, es explicable atendiendo a las variables sociales. De esta forma, se refuerza la idea de que la brecha digital es un tipo más de desigualdad social al mismo tiempo que se pone de manifiesto que las políticas de reducción de la brecha digital podrían aumentar sus resultados de orientarse hacia este tipo de diferencias y no, tal y como se produce en este momento, hacia las clasificaciones geográficas y geográfica-políticas de los diferentes territorios.

\section{BIBLIOGRAFÍA}

Agresti, A. (1990): Categorical Data Analysis. Chichester: John Wiley \& Sons.

ANderson, R. H., BiKson, T. K., LAW, S. A., Mitchell, B. M. (2001): Universal access to e-mail: feasibility and societal implications. en: Compaine, B. M. (Ed.), The digital divide. Facing a crisis or creating a myth? MIT Press, Cambridge, MA, pp. 243262.

BIMBER, B, (2000): «Measuring the gender gap on the Internet». Social Science Quarterly $81,868-876$.

BonfADELLI, H, (2002): «The Internet and knowledge gaps. A theoretical and empirical investigation». European Journal of Communication 17, 65-84.

BuCY, E. P. (2000): «Social access to the internet». Harvard International Journal of Press/Politics 5, 50-61.

CAstells, M. (2005): La era de la información, vol. I «La sociedad red», Alianza, 3. a ed., Madrid (p.o. 1998).

Compaine, B. M, (2001): Declare the war won. En: Compaine, B. M. (Ed.), The digital divide. Facing a crisis or creating a myth? MIT Press, Cambridge, MA, pp. 315-335.

Dimaggio, P., Hargittai, E., Neuman, W. R., Robinson, J. P. (2001): «Social implications of the Internet». Annual Review of Sociology 27, 307-336.

Gunkel, D. J. (2003): «Second thoughts: toward a critique of the digital divide». New Media \& Society 5, 499-522.

MAJó, J (1997): Chips, cables y poder: la clase dominante en el siglo XXI. Planeta, Barcelona.

Negroponte, N., (1995): Being Digital. Hodder \& Stoughton, London.

NORRIS, P, (2001): Digital divide? Civic engagement, information poverty and the Internet worldwide. Cambridge, Cambridge University Press.

Jovell, A. J. (1995): Análisis de Regresión Logística. Madrid: CIS.

KnOKe, D. y BuRKe, P. J. (1980): Log-linear Models. Beverly Hills: Sage.

Peter, J. y VAlkemburg, P, (2006): «Adolescents' Internet use: testing the disappearing digital divide versus de emerging digital differentiation approach». Poetics. Artículo en imprenta.

PNUD (2001): «Las transformaciones tecnológicas de hoy: la era de redes» en Poner el adelanto tecnológico al servicio del desarrollo humano, versión online en http://www.revistainterforum.com/espanol/articulos/111201 tecno.html.

RAMONET, I. (2004): «El nuevo orden Internet». Le Monde Diplomatique, año VII, n. ${ }^{99}$, enero 2004. 
RAND (1995): «Conclusions and recommendations». Universal access to e-mail feasability and social implications, versión online en http://www.rand.org/pubs/monograph_reports/MR650/index.html.

RoE, K., Broos, A, (2005): Marginality in the information age: the socio-demographics of computer disquietude. A short research note. Communications 30, 91-96.

RoBLES, J. M. (2005): La Sociedad de la Información en Andalucía. Sevilla, Junta de Andalucía.

RoBLES, J. M (2006): Los jóvenes y las nuevas formas de participación política a través de Internet. En: María Jesús Funes, Jóvenes y la participación. INJUVE.

RoBles, J. M. (2006): Las creencias cuentan: el papel de la percepción de la utilidad de las TIC en el desarrollo de la Sociedad de la Información. En: Diego Becerril. TIC y Sociedad en el siglo XXI. Universidad de Granada.

RuIZ-MAYA, L. (1990): Metodología estadística para el análisis de datos cualitativos. Madrid: CIS.

Seber, G. A. F. (1984): Multivariate observations. Chichester: John Wiley \& Sons.

SELWYN, N. (2004): «Reconsidering political and popular understandings of the digital divide». New Media and Society 6, 341-362.

Silva, L. C. y BARroso, I. M. (2004): Regresión Logística. Madrid: La Muralla.

VAN DiJK, J., (1999): The Network Society. Sage, London.

VAN DiJK, J., (2002): «A framework for digital divide research». Electronic Journal of Communication $12(1,2)$.

VAN DiJK, J., HACKER, K., (2003): «The digital divide as a complex and dynamic phenomenon». The Information Society 19, 315-326.

Walsh, E. O., Gazala, M. E., Ham, C, (2001): The truth about the digital divide. En: Compaine, B. M. (Ed.), The digital divide. Facing a crisis or creating a myth? MIT Press, Cambridge, MA, pp. 279-284. 


\title{
RESUMEN
}

Con la aparición de las Tecnologías de la Información y la Comunicación (TIC) han surgido nuevas formas de desigualdad social. Este es el caso de la brecha digital, la cual hace referencia al desigual acceso a las tecnologías entre diversos grupos sociales por causa, entre otras razones, de su lugar de residencia, su edad o su nivel educativo. Sin embargo, pese al importante aumento de los estudios sobre este fenómeno social, aún desconocemos importantes aspectos de la brecha digital. En este trabajo pretendemos colaborar en el esclarecimiento de algunos de estos aspectos mostrando la importante relación existente entre la brecha digital y las diferencias sociales que caracterizan un determinado territorio. Nos centraremos en el caso andaluz para, a partir de un modelo metodológico estadístico diseñado específicamente para este propósito, defender que las diferencias en el uso de Internet entre unos territorios y otros son plenamente explicables apelando a la desigual distribución social existente entre dichos territorios. Esto nos permitirá concluir, con autores como Van Dijk (2002), que la reducción de la brecha digital está supeditada a la disminución de las diferencias sociales.

\section{PALABRAS CLAVE}

Sociedad de la Información, Brecha digital, estructura social.

\begin{abstract}
The appearance of the Information and Communication Technologies has generated new forms of inequality. This is the case of the digital divide, which refers to the unequal access to the technologies by different social groups due to, among other reasons, their place of residence, their age, or their educational level. However, despite of the growth of the number of studies about this social phenomenon, we are still unaware of important aspects of the digital divide. In this paper we try to contribute to the explanation of some of these aspects showing the relationship between the digital divide and the social differences that characterizes some territory. From a statistical model, specifically designed for this purpose, we will defend that the differences in the use of Internet among different territories can be completely explained resorting to the unequal social distribution among these territories. This will lead us to conclude, with authors like Van Dijk (2002), that the reduction of the digital divide is conditioned to the decrease of social differences.
\end{abstract}

\section{KEY WORDS}

Information society, Digital gap, social structure. 